tide bond; as a donator of the sulphydril groups, cystein was applied $\left(2 \times 10^{-3}\right)$.

Our experiments, carried on in a large number of replications, led to the following conclusions. The clearly pronounced inhibitory effect on rhythmical contractions of the heart muscle, due to the action of the vagus nerve and of acetylcholine, was found to be removed after the application of mercury bichloride solution, that is, after binding the sulphydril groups; as a rule, the effect was restored after the heart was washed with cystein solution. In control experiments, no similar action could be obtained with Ringer or cystein solution.

When the mercury bichloride solution was introduced against a background of the influence of acetylcholine, that is, when the contractile substrate and the corresponding chain of enzymo-chemical processes was subjected to the influence of acetylcholine, but the phase of restoration had not yet begun, the mercury bichloride solution was no longer able to cause the effect recorded by us : after the normal height of the contractions has been restored, every application of acetylcholine was followed by a typical effect of inhibition of the rhythmical contractions of the heart. This effect vanished as a rule in all experiments in which mercury bichloride was applied against a background of normal contractions.

Preliminary experiments have shown that, unlike the effect of mercury bichloride, the well-known effect of elimination of the action of acetylcholine with atropine cannot be restored with cystein, which points to a peculiar biochemical mechanism underlying the action of atropine.

Experiments in this direction are in progress.

Ch. S. Koschtojanz

T. M. TURPAJEW

Department of Comparative Physiology, Institute of Evolutionary Morphology,

Academy of Sciences of the U.S.S.R., Moscow. Sept. 13.

${ }^{1}$ Astbury, W. F., J. Chem. Soc., 337 (1942).

2 Anson, M. L., "Adv. in Prot. Chem.", 2, 363 (1945).

3 Barron, E. S. F., “Adv. in Enzym.”, 3, 177 (1943).

'Nachmanson, D., and Lederer, E., Bull. Soc. Chim. Biol., 21, 797 (1939).

s Koshtojanz, Ch. S., C.R. Acad. Sci. U.R.S.S., 19, 4 (1938) ; 43, 8 (1944).

\section{Specific Action of Optical Isomers of Mepa- crine upon Dextral and Sinistral Strains of Bacillus mycoides Flügge}

THE normal colonies of Bacillus mycoides Flügge growing on the surface of agar medium have filaments with an anti-clockwise spiral and should be called sinistral or $L$-forms; there occur also as very rare exceptions colonies with a clockwise spiral (dextral or $D$-forms).

Our cultures (two $D$-strains and two $L$-strains) were obtained from Prof. E. N. Mishustin (Institute of Microbiology, Academy of Sciences of the U.S.S.R.). Three of them were isolated from soils collected in the vicinity of Lake Sewan, Armenia. Optical isomers of mepacrine hydrochloride were prepared at the Institute of Malaria and Parasitic Diseases according to the method of Chelinzev and Ossetrova ${ }^{1}$. Bacillus mycoides was cultivated on a medium with 3.5 per cent of German agar in a potato broth (200 gm. of potatoes boiled for $30 \mathrm{~min}$. in 1,000 c.c. of water). Mepacrine was added to the hot medium, which was again heated on the following day up to boiling point in a water-bath, or autoclaved. Throughout all experiments (with one exception) 0.01 per cent of mepacrine in the medium was used. This concentration causes approximately a 30 per cent depression of colony growth. Each 10-cm. Petri dish received four point inoculations. The diameter of the colonies was measured after $24,48,72$ or 120 hours from the beginning of growth, by means of dividers. As a measure of the relative toxicity of dextro- and lævo-rotatory mepacrine an index $(d / l) 100$ was taken, where $d$ represents the average diameter of four colonies grown on a medium with $d$-mepacrine, and $l$ that of colonies grown in the presence of $l$-mepacrine.

Seven experiments were carried out. Omitting the third experiment, in which there were some defects in the preparation of the medium, in all experiments the index for dextral colonies was lower than for sinistral ones $(74 \cdot 4$ as against $114 \cdot 1$ per cent). The difference between the indices is statistically significant, being equal to $6 \cdot 51$ with five degrees of freedom. A contingency table for the direction of the spiral and the value of the index lying below and above 100, using the Yates-Fisher adjusted formula, gave $\chi^{2}=17 \cdot 15$, with a probability much below $0 \cdot 01$. This can be considered as a proof of the existence of a relation between the direction of the spiral and of the value of the index. We may conclude that the two forms of $B$. mycoides are characterized not only by an inversion of colonial morphology but also by an inversion of some mepacrine 'receptors' on a molecular level.

According to previous investigations in our laboratory all living organisms, beginning with normally spiralized or $L$-forms of $B$. mycoides and Infusoria up to higher Vertebrata ${ }^{2}$, show an index greater than 100. This means that their living matter is less sensitive to $d$-mepacrine than to $l$ mepacrine. The only exception to this rule known at present is the dextral form of Bacillus mycoides Flügge, which evidently originated by mutation from the normal sinistral form. Gause ${ }^{3}$ succeeded in showing that the $D$-form of $B$. mycoides contains an enzyme which can split unnatural dipeptides (of dextral steric series). In connexion with this, one must keep in mind that Kögl and Erxleben ${ }^{4}$ have isolated from malignant tumours the unnatural isomer of glutamic acid (of dextral steric series), and Waldschmidt-Leitz and Mayer ${ }^{5}$ have discovered in the blood serum of patients with malignant tumours enzymes of unusual stereochemical structure. Taking all these facts into consideration our experiments with $D$ - and $L$-strains of $B$. mycoides perhaps represent a first step towards developing a rational cancer therapy utilizing optical isomers. Drugs similar to $d$-mepacrine inhibiting the growth of cells containing substances with abnormal molecular configuration more strongly than that of cells with normally configurated molecules might perhaps be useful in depressing malignant cell-growth in the animal and human body.

This work was carried out in collaboration with my assistant, Mrs. O. C. Nastukova.

Institute of Zoology,

University of Moscow. ${ }^{1}$ Chelinzev, G. V., and Ossetrova, E. D., J. Gen. Chem., 5, 10 (1940)
(in Russian).

2 Gause, G. F., and Alpatov, V. V., C.R. Acad. Sci. U.S.S.R., 32 , No. 7 (1941) (in English).

'Gause, G. F., Biochemistry, 7 , No. 1-2 (1942) (in Russian).

‘ Kठ̈gl, F. and Erxleben, H., Z. physiol. Chem., 261 (1939).

'Waldschmidt-Leitz, E. K., and Mayer, K., Z. physiol. Chem., 262 (1939). 